\title{
Fully portable, highly flexible dilution refrigerator systems for neutron scattering
}

\author{
P. A. Hilton and N. W. Kerley \\ Oxford Instruments, Osney Mead, Oxford, U.K.
}

\begin{abstract}
Résumé. - Ce papier décrit des systèmes cryogéniques à dilution conçus spécialement pour les expériences de diffusion des neutrons. Les cryostats sont maniables et laissent une grande liberté pour l'environnement de l'échantillon. Deux ensembles de dilution à chargement par le haut sont présentés. Dans le premier, qui permet de réaliser de très basses températures de l'ordre de $6 \mathrm{mK}$, les échantillons sont montés dans le vide. Pour le second, qui atteint une température limite légèrement supérieure de $35 \mathrm{mK}$, les échantillons sont montés dans un boîtier rempli de $\mathrm{He}^{4}$ superfluide.
\end{abstract}

\begin{abstract}
This paper describes dilution refrigerator systems developed specifically for neutron scattering environments. The refrigerators are completely portable, and yet still retain a large degree of flexibility regarding sample environment. Two types of top loading dilution unit are described. The first, which offers a very low base temperature in the region of $6 \mathrm{mK}$, allows samples to be top loaded into vacuum. The second, with a higher base temperature of $35 \mathrm{mK}$, allows samples to be top loaded into a container containing superfluid helium-4.
\end{abstract}

Temperatures as low as 3 to $5 \mathrm{mK}$ are now routinely available in many research laboratories by using continuously operating dilution refrigerators. Until relatively recently however, the lowest temperatures available in almost all neutron scattering laboratories have been in the region of $1.2 \mathrm{~K}$. One reason for this is the relative complexity of the equipment needed to work continuously at temperatures below $1 \mathrm{~K}$. This sort of equipment has been regarded by many scientists working in the field of neutron scattering as being so complex, as to be by definition, unreliable. The requirement for efficient use of the available beam time in neutron scattering laboratories, has therefore kept the experiments performed in the temperature regime below $1 \mathrm{~K}$ to a minimum, even though many interesting experiments requiring millikelvin temperatures and neutron scattering techniques have been proposed. The most convenient method of producing temperatures below $1 \mathrm{~K}$ for use in neutron scattering experiments is by employing a dilution refrigerator. This paper describes how the «laboratory " dilution refrigerator has been developed for use in the environment of a neutron scattering laboratory.

For reasons of experimental flexibility, the refrigerator must be designed so that it can be used on several spectrometers. This effectively means that the cryostat must be separate from the pumping and gas handling systems. The cryostat must also have a degree of rotational freedom when mounted on the spectrometer table, (say $\pm 50^{\circ}$ ) without moving the pumping cabinet. As a result of this, the interconnecting lines between cabinet and cryostat must be flexible and relatively long. In addition to these basic requirements, additional features are required if a magnetic field is to be included in the sample region, or if it is required that samples may be interchanged while the refrigerator is operational. The dilution refrigerator should also have a high cooling power, so that warming due to vibrational heat leaks is kept to a minimum and top loaded samples can be cooled to low temperatures quickly.

The whole system must also be designed so that operations such as top loading and moving from spectrometer to spectrometer can be performed with the minimum amount of labour. In fact, even the most complicated operations with these refrigerator systems can be accomplished using a maximum of 2 people. The requirement of high cooling power, which requires a large pumping system and short, large diameter pumping lines, conflicts somewhat with the need for a compact, mobile pumping system with the cryostat remote from the cabinet. The resulting refrigerator system is therefore a compromise and 
consists of a cryostat, approximately $40 \mathrm{~cm}$ diameter and $160 \mathrm{~cm}$ high, connected to a control cabinet by a set of 6 flexible pumping lines $3 \mathrm{~m}$ long. The cryostat has been designed so that the neutron beam passes only through pure aluminium windows. For ease of manipulation, the diameter of the helium-3 pumping line has been kept to $5 \mathrm{~cm}$.

The remaining vacuum lines are each $2.5 \mathrm{~cm}$ diameter. Included in the cabinet, which is approximately $2 \mathrm{~m}$ high and $1 \mathrm{~m}$ square, are the full vacuum, security and gas handling systems needed for operation of the refrigerator. When not in use and during precooling, the cryostat can be mounted onto a support at the rear of the cabinet. Thus, it is never necessary to separate the flexible pumping lines between the cryostat and the cabinet. The cabinet can be moved either on wheels or by overhead crane.

An important feature of the refrigerator is the helium-3 circulation system. The cooling power of the dilution unit is dependent on the helium-3 circulation rate. As only modest pressures are needed to keep the still cold, much higher circulation rates are available by using an oil vapour booster pump to circulate the He-3 rather than a diffusion pump. The booster pump also requires a much smaller backing pump than a comparable diffusion pump. The pumping system chosen for this application is an Edwards 9B3 booster pump, backed by a helium-3 sealed $30 \mathrm{cu} \mathrm{m} / \mathrm{h}$ rotary pump. Both pumps are included within the cabinet. The maximum $\mathrm{He}-3$ circulation rate available with this combination of pumps (while pumping through the long flexible line to the still) is 450 micromoles/s, with a still pressure of about 0.1 mbar. This high circulation rate minimises the cooldown time of the dilution refrigerator.

The system described above can be used with two types of top loading dilution unit. The first type employs a dilution unit incorporating a tubular, counterflow heat exchanger, followed by 4 sintered silver heat exchangers. Samples may be top loaded into a screw thread attached to the mixing chamber of the dilution unit. The dilution unit is shown in figure 1. This system is designed to operate with a 5 tesla split pair magnet and thus the sample must be loaded into a position within the split of the magnet. A main feature of the dilution unit is the central access tube, which allows a sample up to $10 \mathrm{~mm}$ in diameter to be loaded into the refrigerator. This central access can be opened by moving a sliding radiation baffle across the plate attached to the still. A second radiation baffle operates at $4.2 \mathrm{~K}$. Both radiation baffles are operated from room temperature. The thermal path from the sample to the mixing chamber is provided by a series of copper to copper screw threads. Provided the threads are kept reasonably clean, a simple screw thread is a very effective joint at low temperatures. At Oxford Instruments temperatures as low as $3.5 \mathrm{mK}$ have been measured using this type of joint. Top loading is achieved by pre-cooling the

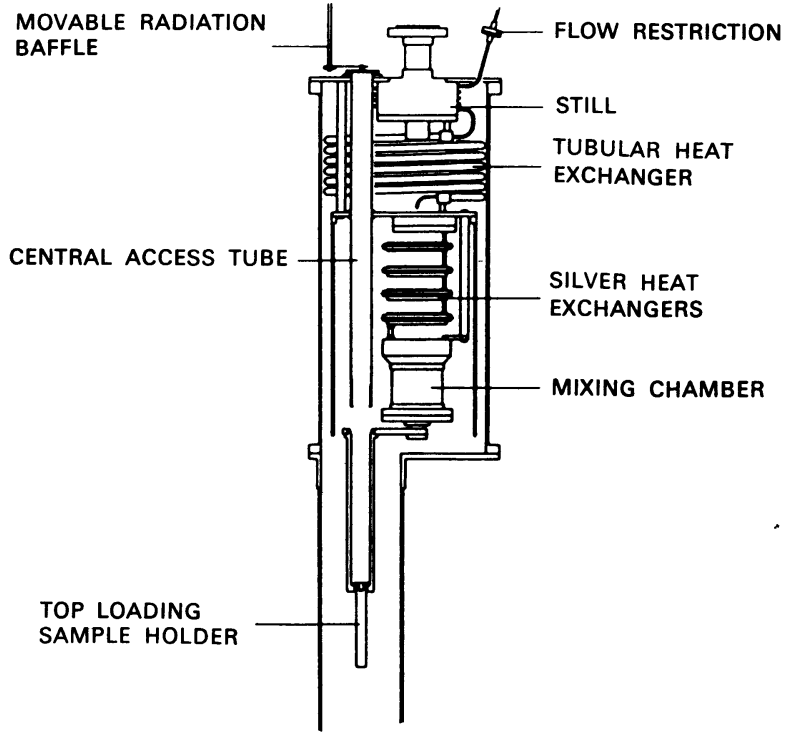

Fig. 1.

sample to $4.2 \mathrm{~K}$ using a special type of transfer tube. This pre-cooling takes place within the vacuum of the central access of the refrigerator. The sample at $4.2 \mathrm{~K}$ is then quickly transferred to the mixing chamber using the transfer tube as a removable screwdriver. During this process the mixing chamber warms up to about $1.5 \mathrm{~K}$ and then cools rapidly, as the dilution unit takes over. A schematic drawing of the various components needed for top loading is shown in figure 2. The samples are mounted onto a copper rod which is screwed to the special $T$ shaped top loading siphon using a left handed thread. The siphon is lowered through a vacuum lock into the working dilution refrigerator, to a position just above the $4.2 \mathrm{~K}$ movable baffle. The sample is now cooled to $4.2 \mathrm{~K}$ by connecting one arm of the top loading siphon to a helium storage dewar and drawing helium through the top loading siphon. Pre-cooling takes typically 15 to 20 minutes.

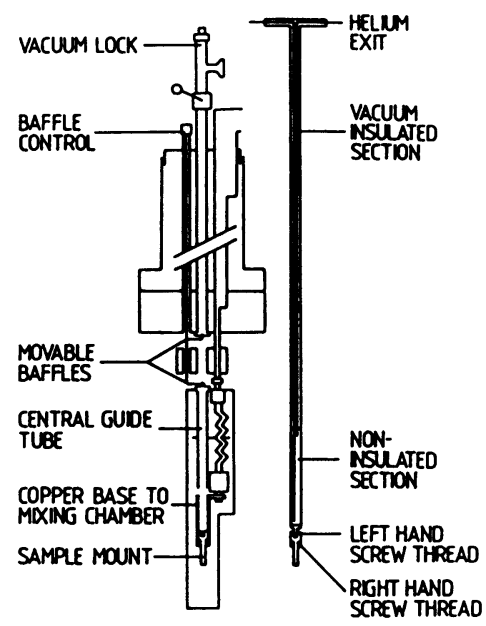

Fig. 2. - Top loading into vacuum. 
The top loading siphon is then disconnected from the storage dewar, the movable baffles are opened and the sample at $4.2 \mathrm{~K}$, is lowered through to the mixing chamber. The siphon is then rotated clockwise until the sample is screwed tightly into the mixing chamber. At this point the left hand thread breaks and the siphon is rotated further until it can be withdrawn, thus leaving the sample mechanically and thermally transferred to the mixing chamber.

During testing, the base temperature of the sample after top loading was measured at $5.6 \mathrm{mK}$ and the samples can be cooled to below $10 \mathrm{mK}$ in less than 3 hours. For these measurements, the thermometer was mounted onto the top loading sample holder, thus simulating actual experimental conditions as closely as possible. Temperatures were measured using gamma-ray anisotropy from radioactive cobalt 60 within a cobalt single crystal.

The second type of dilution unit to be described, allows samples up to $20 \mathrm{~mm}$ diameter to be top loaded directly into an aluminium cell suspended below the mixing chamber and containing liquid helium-4. For a neutron scattering experiment, it is important that any helium-3, which has an exceptionally high absorption cross section, is kept out of the beam path. This dilution unit is shown in figure 3. The liquid helium-4 is kept in thermal contact with the helium-3/ helium- 4 mixture within the dilution unit by means of sintered silver sponges. The helium-3/4 mixture in the dilution unit is completely separate from the helium-4 in the sample space. This dilution unit employs only a single counter flow heat exchanger, the first stage of which is wound directly onto the central tube containing the helium-4. Samples, which

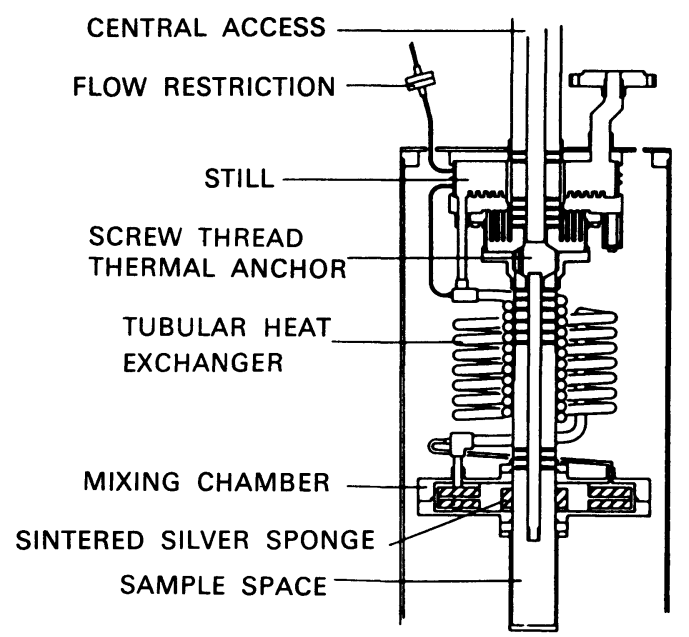

Fig. 3. - Top loading dilution unit sample in helium-4. in this case may also be powders, are introduced through a vacuum lock into the central access tube. Several thermal contacts, typically at $0.6 \mathrm{~K}, 1.5 \mathrm{~K}$ and $4.2 \mathrm{~K}$ anchor the support rod for the sample and also provide mechanical alignment.

The main design problems with this type of dilution unit are associated with the superfluid film flow from the helium-4 within the central tube. In order to keep the heat leak due to this film between the mixing chamber and the still to a minimum, the counterflow heat exchanger is wound directly onto the central tube. It is also vitally important that the heat leak due to the evaporated film recondensing, does not reach the mixing chamber. Given the perimeter of the tubes concerned, this heat leak has been estimated at a minimum of $2 \mathrm{~mW}$. It is clear that this is far too much for the mixing chamber alone to absorb. The design of the dilution unit attempts to force the evaporated film to re-condense at still level, by providing a large surface area at the temperature of the still and only a very small diameter hole in the threaded thermal anchor situated just below the still. The sample support rod is assembled with several copper baffles providing a close fit to the central tube.

Tests were performed using sufficient liquid helium4 in the sample can to fill the system up to the level of the sintered silver ring at mixing chamber level. With the dilution unit operational and no external heat input to the still, the minimum helium-3 flow was 270 micromoles/second. When the centre tube was completely empty of helium-4, the still needed to be energised with $3 \mathrm{~mW}$ of electrical power to promote the same helium-3 flow. This figure of $3 \mathrm{~mW}$ can be compared to the estimated $2 \mathrm{~mW}$ heat load provided by the re-fluxing helium- 4 film. The fact that the dilution unit did not require additional electrical stimulation to maintain a high helium-3 flow, is evidence that a large proportion of the heat leak caused by the re-condensing helium- 4 film is absorbed at the still level. The nuclear orientation thermometer was positioned on the end of a copper spigot protruding through the base of the sample can. Onto the top end of the rod was a quantity of sintered silver. The continuous base temperature recorded was $35 \mathrm{mK}$, at the minimum helium-3 circulation rate of 270 micromoles per second. The cooling power of the dilution unit was greater than 100 microwatts at $100 \mathrm{mK}$.

The development of these dilution refrigerator systems, has shown that experiments in the millikelvin regime are no longer limited to specialist low temperature laboratories. 